Mots. Les langages du politique

\title{
La stratégie de l'évanouissement. À propos des tempêtes de décembre 1999
}

Claude Jamet

\section{(2) OpenEdition \\ Journals}

Édition électronique

URL : https://journals.openedition.org/mots/3393

DOI : $10.4000 /$ mots.3393

ISSN : 1960-6001

Éditeur

ENS Éditions

Édition imprimée

Date de publication : 1 juillet 2004

Pagination : 77-87

ISBN : 2-84788-057-7

ISSN : 0243-6450

Référence électronique

Claude Jamet, "La stratégie de l'évanouissement. À propos des tempêtes de décembre 1999 », Mots Les langages du politique [En ligne], 75 | 2004, mis en ligne le 22 avril 2008, consulté le 23 avril 2022. URL : http://journals.openedition.org/mots/3393 ; DOI : https://doi.org/10.4000/mots.3393 


\section{La stratégie de l'évanouissement. À propos des tempêtes de décembre 1999}

L'analyse du discours «peut tenter d'étudier le processus discursif par lequel l'émotion peut être mise en place, c'est-à-dire traiter celle-ci comme un effet visé (ou supposé), [mais] sans jamais avoir de garantie sur l'effet produit», fait remarquer Patrick Charaudeau ${ }^{2}$. C'est ce qui sera tenté ici : analyser un procédé discursif qui essaye de rendre compte de l'émotion soulevée par les tempêtes de décembre 1999. Leur violence, leur durée, leur répétition deux jours de suite, leur extension à la quasi-totalité du territoire font de cet évènement un prototype a priori source d'émotions. Or, pour traiter un tel sujet, deux hebdomadaires et un supplément spécial de la presse écrite française font un choix discursif identique : renoncer à tout récit bouclé ${ }^{3}$ du météore.

Cette curieuse «coïncidence» est à l'origine de notre corpus. Il est composé des livraisons parues entre le 6 et le 13 janvier 2000 du Nouvel Observateur et de L'Express, et du supplément du Monde en date du 13 janvier, exclusivement consacré à l'évènement sous le titre de «La tempête des tempêtes».

En refusant toute construction narrative (ou dramatique, si l'on parle comme Aristote) complète, le discours journalistique parait à première vue esquiver les risques de dramatisation (au sens courant du terme), source facile de l'émotion. Pourtant, il nous semble que ce refus de raconter ce qui s'est passé favorise le déploiement d'une stratégie émotionnelle: la mise en scène discursive de l'inaptitude apparente des signes (linguistiques et iconiques) à rendre compte d'un évènement est une autre façon, en réalité, de suggérer l'émotion.

1. Institut de la communication - Université Lumière, Lyon 2 - claude.jamet@univ-lyon2.fr

2. P. Charaudeau, 2000, «Une problématisation discursive de l'émotion. Àpropos des effets de pathémisation à la télévision », C. Plantin, M. Doury et V. Traverso (dir.), Les émotions dans les interactions, Lyon, PUL, p. 125-155.

3. Le récit, ou drame, dit Aristote, est une histoire construite «autour d'une action une, formant un tout et menée jusqu'à son terme, ayant un milieu, un commencement et une fin, pour que pareille à un être vivant qui est un et forme un tout, elle procure le plaisir qui lui est propre». Aristote, 1990, Poétique, Paris, Le Livre de poche, p. 112. 


\section{Le refus de la narration}

Une première façon de jouer de l'émotion réside dans cette suggestion du caractère indicible de l'évènement: tout est trop violent, démesuré, sans référent historique... pour que l'on ait «les mots pour le dire». Alors que le lecteur pourrait s'attendre à un récit circonstancié, il constate cette absence paradoxale, l'évanouissement du fait. La mise en scène adoptée par ces trois journaux est construite autour de cet évanouissement.

Si L'Express expédie le récit de la tempête en une dizaine de lignes (organisées autour de participes présents) dans un article qui en compte plus de six cents - une façon encore relative de gommer l'évènement - les deux autres journaux sont plus radicaux.

Le dispositif du supplément spécial du Monde est le plus extrême. À l'image de la une, entièrement occupée par deux photographies titrées l'une "Avant», l'autre «Après », chacune des huit pages s'organise autour du même couple: un paysage approximativement identique pris l'un avant, l'autre après la tempête. On ne trouve aucun cliché de la tempête elle-même. Ce fonctionnement iconographique, qui exclut spectaculairement l'objet dont le supplément prétend rendre compte, a son équivalent dans l'écrit: la seule relation des évènements se trouve dans une chronologie succincte (dix-huit lignes sur les huit pages), non pas des faits d'ailleurs, mais des alertes météo (vitesse des vents). Quand le papier s'aventure dans le récit, il court-circuite le segment narratif concernant la tempête ellemême. Les hiatus du temps «bricolent» ainsi une curieuse bâtisse narrative, comme inachevée, dont l'imaginaire du lecteur aurait à combler les béances.

L'article du Nouvel Observateur procède de façon similaire. Il s'ouvre sur une indication de temps et l'identification d'un actant, inauguration discursive qui marque habituellement une organisation narrative forte. Dimanche 26 décembre, vers six heures du matin, Patrick Novello s'est réveillé inquiet. Alors qu'il prend son petit déjeuner, il voit les deux épicéas de son voisin s'abattre sous les rafales. Employé de l'ONF, il saute dans sa voiture et fonce «vers ses forêts d'Ile-deFrance dont il a depuis dix-huit ans la charge ». Paragraphe suivant, Patrick Novello arrive dans sa forêt: «Partout c'est le désastre. Jamais vu un tel massacre. Patrick en pleurerait de rage et de désespoir. C'est son travail de plusieurs années qui vient d'être balayé...». Le paragraphe se poursuit par la description de la forêt dévastée et s'achève sur ces deux phrases: "Troncs éclatés, arbres foudroyés, souches et racines dressées vers le ciel, branches enchevêtrées... Deux jours après, la forêt semble encore avoir été le théâtre d'une bataille de titans ». Le récit mort-né est abandonné: ce qui est proposé au lecteur n'est qu'une ébauche de récit marquée, de plus, par trois lacunes temporelles. 
La première se trouve, suggérée, dans le mot désastre, dont on sait qu'il admet une composante sémantique orientant le sens, depuis l'acception première d'évènement funeste, vers les conséquences de cet évènement. Cette interprétation est confirmée par un deuxième indice: l'auxiliaire d'aspect vient d'être marque grammaticalement la fin d'un procès dont on souligne la proximité immédiate dans le passé. Reprenant le fil du discours journalistique, on constate que le voyage du témoin, de chez lui à sa forêt, n'est pas raconté. Or, ce déplacement spatial ignoré par le récit coïncide exactement avec la durée de la tempête. En effet, parti quand cette dernière débute, Patrick Novello arrive lorsque la forêt «vient d'être balayée ». Comme fréquemment dans un récit, à un saut spatial sur un vecteur orienté correspond une ellipse temporelle.

À la fin du paragraphe, le lecteur rencontre un dernier hiatus temporel, construit par les points de suspension et l'expression «deux jours après», qui ouvre la dernière phrase. Celle-ci est explicite et parait déporter la suite de l'article loin déjà de la tempête. Cependant, cette reconstruction de la trame du temps reste hypothétique. Deux éléments pourraient en effet suggérer une autre interprétation. La phrase «Deux jours après... » est située à la fin du deuxième paragraphe et non au début du troisième. Elle semble donc se rattacher à celuilà plutôt qu'à celui-ci. Par ailleurs, le troisième paragraphe se poursuit avec des verbes au présent narratif et avec le même acteur, Patrick Novello, dans un rôle similaire à celui qu'on lui imagine, découvrant l'ampleur de la destruction: «Patrick court d'un arbre à l'autre, les touche, grimpe sur les talus». Une telle lecture redistribue les rôles de chacun des éléments discursifs que nous avons relevés. Les points de suspension ne marquent plus une ellipse de temps mais l'inachèvement de l'énumération. Dans cette hypothèse, la dernière phrase peut se comprendre comme une conclusion qui cadrerait le temps du paragraphe dans lequel elle se trouve (le deuxième). Par ailleurs, les caractéristiques des temps verbaux et l'attitude de l'agent forestier dans le troisième paragraphe sont à considérer dans la continuité temporelle et logique du paragraphe qui le précède. En d'autres termes, l'hiatus temporel de deux jours ne serait plus à situer à la fin du deuxième paragraphe mais au début... Relisons : quand Patrick Novello, au début du deuxième paragraphe («Partout, c'est le désastre»), arrive sur les lieux, deux jours se sont écoulés depuis la tempête, et non les deux ou trois heures nécessaires au trajet.

Il est pourtant impossible de décider en faveur de cette hypothèse contre la précédente. Cette difficulté renvoie à un constat souvent souligné ${ }^{4}:$ la structure temporelle des articles de presse est ordinairement complexe. Cependant, ce

4. C. Jamet et A.-M. Jeannet, 1999, Les stratégies de l'information, Paris, L'Harmattan, p. 19 et suiv. 
que cache la complexité nous intéresse plus que la complexité elle-même. Cette ellipse serait à ranger dans une curieuse catégorie que nous serions tentés de confectionner à partir des propositions de Gérard Genette 5 . Il s'agirait d'une ellipse explicite hypothétique; explicite parce qu'il est dit que deux jours se sont écoulés, hypothétique parce que cette ellipse est impossible à localiser. L'ellipse est ainsi à double effet. Non seulement elle tait l'évènement, uniquement évoqué par ses prémices (la chute des épicéas) et par son positionnement comme temps relatif (à travers l'expression «deux jours après»), mais elle rend incertaine la chronologie du récit. Or, comme de toute incertitude, il sourd également de celle-ci un filet d'inquiétude qui ajoute à la crainte née de l'effacement narratif de l'ouragan. Ainsi la tempête disparait dans une sorte de «trou noir» du récit qui, parallèlement, l'estompe dans le non-dit et la suggère dans les interstices marqués de la trame narrative, mais à une place temporelle indéterminable, comme librement suspendue dans la chronologie, en quelque sorte à la libre disposition de l'imagination du lecteur.

Cette manipulation discursive - effacement et indistinction chronologique renvoie à une des caractéristiques de la crainte qui, de toutes les émotions, est ici principalement convoquée. En effet, si l'émotion est définie comme la réaction à une situation déstabilisatrice, avec pour finalité de rétablir une certaine façon d'être-au-monde, la réaction à la peur serait la négation de l'objet qui l'engendre. C'est ce que suggère Jean-Paul Sartre que nous suivrons pour l'occasion:

Le véritable sens de la peur nous apparait: c'est une conscience qui vise à nier, à travers une conduite magique, un objet du monde extérieur et qui ira jusqu'à s'anéantir [par exemple, par l'évanouissement], pour anéantir l'objet avec elle ${ }^{6}$.

«Conduite magique», parce qu'elle supprime l'objet en tant qu'il est objet de conscience et non, bien sûr, en tant qu'il est objet du monde. Procédant à la mise en scène discursive de la disparition de l'objet même de l'article (mais de façon telle que l'on n'ignore rien de sa réalité), le journaliste ne cherche pas à nier la tempête. Ne choisit-il pas plutôt de décalquer la conduite magique opérée par la conscience sous le coup de la peur? Quand il laisse penser qu'il n'a pas «les mots pour le dire», ne signifie-t il pas que cette impuissance est une fuite dans le mutisme de la même façon que, dans la réalité, l'impuissance à agir, due à la surcharge émotionnelle, peut conduire à la néantisation magique de l'objet que l'on craint? Son discours réintégrerait ainsi, par similarité mutique, la proximité émotive avec l'évènement. Paradoxe: c'est en quelque sorte en la déréalisant que le journaliste rend à l'évènement sa pleine réalité émotionnelle

5. G. Genette, 1972, Figures 3, Paris, Seuil, p. 139 et suiv.

6. J.-P. Sartre, 1961, Esquisse d'une théorie des émotions, Paris, Hermann, p. 46. 
car il laisse ainsi au lecteur une place discursive disponible pour que ce dernier l'occupe émotionnellement, au-delà - ou en-deçà - des mots.

\section{La mise en scène discursive de l'absence}

Cette interprétation ne tient que parce qu'elle trouve dans le discours des indices qui la confortent. Le journaliste signale cette disparition de la même façon qu'un trou noir donne des preuves de son existence: on le devine aux marges à des phénomènes que sa présence seule peut expliquer. C'est ce qui est le plus explicitement fait avec la série de couples photographiques du Monde titrés «Avant»/ «Après ». Cette «signalétique discursive» ne se limite pas à suggérer l'existence de l'objet absent, elle oriente la façon de le construire. Dans la stratégie de l'effacement, l'activité prédicative du discours est essentielle.

La visée émotive se manifeste donc à travers la conjonction de trois lieux différents du discours: le mutisme sur les tempêtes, l'indication des limites (dessinées par défaut) de l'objet tu, enfin les indices propres à orienter l'imaginaire du lecteur dans «la bonne direction».

Si le récit de la tempête est abandonné, l'objet lui-même garde une certaine matérialité, en premier lieu à travers le paratexte. A contrario, ce dernier est beaucoup plus explicite que les articles: «Climat, que se passe-t-il?» (L'Express), «La tempête des tempêtes» (Le Monde), «Tempête, l'homme a-t-il changé le climat?» (Le Nouvel Observateur). Ces titres ont deux fonctions : nommer ce que les articles taisent et procéder à un élargissement thématique.

Ces trois titres pourraient s'inscrire dans la logique des magazines d'information: la prise de distance par rapport à «l'actualité chaude» se traduit par un élargissement du propos. Mais, dans cet élargissement, il n'y a pas que de la rationalisation (id est sortir le fait de son isolement pour le comprendre dans la causalité), il faut y voir aussi une façon de légitimer l'émotion. En effet, une menace permanente pèse sur cette dernière: l'inauthenticité. Or, fait remarquer Luc Boltanski, «la preuve du caractère inauthentique de l'émotion est qu'elle n'est pas suivie d'action ${ }^{7}$. L'élargissement thématique, qui introduit la responsabilité humaine dans le changement climatique, montre comment le journal assume cette responsabilité en action. De la seule façon qui lui soit permise : en expliquant encore et encore, afin qu'à son tour le lecteur - peut-être - agisse. L'action du journal est d'abord action pédagogique.

Cependant, le titre dit autre chose. Outre qu'il vise la captation, ce qui nous intéresse peu ici, il donne les indices de ce que devra être une «bonne lecture»

7. L. Boltanski, 1993, La souffrance à distance, Paris, Métailié, p. 69. 
des articles. Le titre forme un système discursif où sont posés un thème et un rhème ${ }^{8}$ dont la fonction est de cadrer la lecture. Or, ces trois titres répètent un thème et un rhème identiques:

- ces tempêtes sont un évènement qui va au-delà de l'accidentel (elles touchent au climat ou bien sont l'acmé, provisoire peut-être, d'une série dont on ne prend conscience qu'à cette occasion);

- à ce titre, ces deux ouragans ouvrent des perspectives inquiétantes.

Les trois titres délimitent un thème (le changement climatique dont ces tempêtes ne sont qu'un symptôme) et en proposent une lecture (cela est très inquiétant). En créant une tension diffuse, l'inquiétude forme un arrière-plan à l'émotion. Présent dans la déstabilisation narrative, ce sourd sentiment d'inquiétude l'est aussi dans les titres.

Il se trouve également construit par le déploiement d'isotopies marquées qui, elles aussi, se retrouvent d'un article à l'autre. Le lexique s'organise en deux réseaux qui exploitent l'un la thématique de la démesure, l'autre celle de la violence. S'ajoute, très présent, le réseau subséquent de la crainte. Si l'on considère la seule titraille, le premier réseau accumule les massacre, catastrophe, désastre, apocalypse, cataclysme, violence..., évidemment attendus; et le second: inquiétants phénomènes, évolution troublante, cumul des risques, alertes... Ces réseaux sont transparents et bien peu surprenants. Ils balisent l'inquiétude ordinaire devant la violence incontrôlable de la nature et jouent ainsi un rôle familier en installant le discours dans une aire repérable: la catastrophe naturelle. Leur vocabulaire n'a donc pas seulement pour visée de caractériser une situation donnée, il sert aussi à classer l'article dans une typologie récurrente. Cette généralisation dans une catégorie figée a comme principal intérêt de parler du thème sans avoir à raconter. Mais surtout, elle offre une orientation à la façon dont on doit, en conséquence, l'imaginer.

Le réseau de la démesure cataclysmique présente une autre caractéristique. Il est conventionnellement hyperbolique. Or, fait remarquer Olivier Reboul,

L'hyperbole n'est pas une figure du mensonge, [...] elle est une figure de l'expression [...]. Mais pour exprimer quoi? L'inexprimable sans doute. La fonction sémantique de l'hyperbole est, à notre avis, de dire qu'on ne peut pas vraiment dire, de signifier que ce dont on parle est si grand, si beau, si important (ou le contraire) que le langage ne saurait l'exprimer'.

C'est ce que nous signalions ci-dessus puisque, en réalité, entre ne pas dire et signifier qu'on ne peut pas dire vraiment, il y a modulation discursive et non mutation du sens.

8. M. Mouillaud et J.-F. Tétu, 1989, Le journal quotidien, Lyon, PUL, p. 120.

9. O. Reboul, 1991, Introduction à la rhétorique, Paris, PUF, p. 130. 
La métaphore est une autre façon de suggérer l'absence. «La machine s'emballe», une «main géante» a couché les arbres (Le Monde), «C'est son enfant qui souffre» (Le Nouvel Observateur)... On croit parfois lire du Zola. Or, la transformation métaphorique - qu'on l'entende comme le groupe $\mathrm{Mu}^{10} \mathrm{ou}$ comme Gérard Genette - prend toujours sa source dans « une co-possession partielle, de sèmes et donc [dans une] intersection logique ${ }^{11}$. Mais, fait remarquer le groupe $\mathrm{Mu}$, dans ce mécanisme, qui affecte la totalité du terme explicite, le terme intermédiaire (l'intersection logique) est toujours absent. Au lecteur de ressentir l'essence même de la métaphore: l'absence. Car la métaphore même construite in praesentia est, tout autant que celle bâtie in absentia, marquée organiquement par un absent discursif.

\section{Le discours iconique}

D'où vient que «nous prenons plaisir à contempler les images les plus exactes de choses dont la vue nous est pénible dans la réalité, comme les formes d'animaux les plus méprisés et des cadavres», s'interrogeait Aristote ${ }^{12}$. Ce qui nous intéresse ici n'est pas sa réponse (c'est « une tendance naturelle aux hommes») mais le constat lui-même. Les photos des articles de nos trois journaux présentent deux caractéristiques communes. L'une illustre la remarque d'Aristote, l'autre semble s'y opposer.

On remarque, d'abord et sans surprise, le grand nombre de photos sur les conséquences des deux tempêtes: forêts fauchées, toitures arrachées, pylônes abattus..., accumulation dont, évidemment, «la vue nous est pénible» mais que nous regardons. Cependant, aussi réaliste soit la photo, elle ne peut masquer qu'elle n'est qu'un substitut. Il lui manque toujours quelque chose. Or, ce qu'elle omet est ici particulièrement sensible. Soudainement, et plus que dans tout autre type de photo, le reste de la scène fait défaut: l'odeur, le bruit (ou le silence), l'humidité, le froid, la nuit qui vient vite faute d'électricité..., tout ce que l'imaginaire, sollicité par ailleurs, a commencé à reconstruire des évènements.

Il manque aussi des hommes. Leur présence est accidentelle et, sauf exception, parait «hors sujet». Seules les choses semblent avoir souffert. Si l'homme souffre à ce spectacle, c'est de voir détruites sa forêt, sa maison ou sa voiture. Lui-même est indemne, semble-t il, ou bien alors sa souffrance, physique ou affective, est cachée. L'imaginaire ne peut que travailler sur cette absence; or

10. Groupe $\mathrm{Mu}, 1982$, Rhétorique générale, Paris, Seuil, p. 106 et suiv.

11. G. Genette, 1970, «La rhétorique restreinte», Communications, ${ }^{\circ}$ 16, p. 167.

12. Aristote, 1990, op. cit., p. 89. 
les voies de ce travail ne sont guère nombreuses et en croisent d'autres que nous avons déjà relevées. En effet, soit la photo a pour objet les destructions matérielles - c'est un outil d'expertise, par exemple - et les humains n'y ont pas leur place (les rares que l'on aperçoit semblent bien portants, parfois souriants), soit elle a un autre objet - les destructions en général - et dans ce cas, où sont les victimes humaines et que font ici ceux qui ne semblent pas avoir souffert autrement que dans leurs biens?

Le Monde semble emprunter la première voie. Les couples de photos (Avant / Après) se donnent comme le compte rendu des dégâts matériels, non pas dans leur totalité - inaccessible - mais à travers quelques exemples symboliques : patrimoine forestier, monument historique, cité HLM... Au-delà de l'inventaire, les photos du Monde participent, nous l'avons vu, au jeu discursif transparent par lequel les tempêtes, explicitement annihilées, surmontent leur évanouissement grâce à une évidence construite par l'implicite. L'objet du discours, dans la structure duelle des photos, est ainsi constitué in absentia par les seuls contours de ce qui est donné comme échappant à toute représentation.

Mais la construction de ce discours iconique - une binarité incluant un tiers manquant - est une manière suffisamment curieuse de la photo de presse pour qu'on s'y arrête. La photographie, Roland Barthes insiste constamment sur ce point, est une affirmation de l'avoir-été-là ${ }^{13}$. Aucun doute que les photographies $\mathrm{du}$ Monde répondent à ce constituant existentiel. L'Avant comme l'Après ontété-là, réellement là. Cependant, elles nous disent dans le même temps : il est un avoir-été-là qui n'est pas là, réellement pas là. Le réel du discours (c'est-àdire la matérialité des signes dans le dispositif de la page) met ainsi en scène l'impuissance de ce dernier à rendre compte du «réel tout court». Ce processus est fondamentalement différent de celui, fréquent dans la photo de presse actuelle, qui conduit à la construction de l'absence par une relation indiciaire vers le hors-champ de l'image. Ce type de décalage vers le hors-champ joue du découpage dans le continuum spatial.

Or le système lacunaire du discours iconique construit par Le Monde touche à toute autre chose: au lien indiciaire ontologique avec le temps; c'est-à-dire à ce par quoi la photographie est un système de signes distinguable de tous les autres et ce par quoi, en définitive, elle prétend rendre compte du réel. Ce n'est pas un morceau d'espace qui échappe à l'objectif, c'est un segment du temps qui se dérobe à la photographie. La stratégie de l'évanouissement ouvre ainsi une sorte de gouffre sous les pieds du lecteur: il est du temps, semble-t il être dit, que même la photographie n'a pu saisir. Mais justement, l'émotion n'estelle pas aussi dans cette impuissance à rendre compte du réel?

13. R. Barthes, 1964, «Rhétorique de l'image», Communications, n 4, p. 47. 
Nous glissons ainsi vers la deuxième hypothèse, qui ne peut se construire que par une série de questions. Que cache cette impuissance photographique? Le plaisir du regard dont parle Aristote serait-il à ce point déplacé, voire pervers, qu'il nous soit refusé ? Ou bien la violence des éléments est-elle décidément trop forte pour être représentable sans subir une dégradation telle qu'elle n'en serait que trop affaiblie, et donc mensongère? Est-ce à dire que, pas plus qu'il n'a été possible de trouver «les mots pour le dire», il n'est possible de trouver «les photos pour le montrer»? Et pour quelles raisons? Techniques, déontologiques, morales, fantastiques, ou plus grave encore, pour quelque raison touchant à la nature même de ce qu'on ne peut ni raconter, ni montrer? Il n'est pas de réponses définitives: l'émotion reste dans la boite noire. Nous suggérerons seulement que le coup de force discursif (en tant qu'il sort des règles usuelles de la photographie de presse) a probablement un impact émotionnel pour les mêmes raisons que celles du discours linguistique: l'inquiétude hante le dispositif iconique. Elle nait de l'évanouissement du fait mais aussi et peutêtre plus encore de ce que le signe lui-même est déstabilisé dans sa régularité par la mise en scène discursive.

Opérons un dernier «arrêt sur image», sur la photographie à la une du Nouvel Observateur. Elle occupe la totalité de la page, sans aucune marge, même infime. Le texte (nom du journal, titre, prix...) s'y inscrit même sans espace propre délimité. Le tiers inférieur représente une masse d'eau secouée par des vagues violentes. Les deux autres tiers de la page sont censés représenter un ciel plombé de nuages informes, aux couleurs inhabituelles et qu'un éclair d'orage embrase. Rien ne permet d'apprécier l'échelle de ce paysage. Cette photographie est, à l'évidence, un montage résultant de manipulations numériques: le grain, les couleurs inusitées, la netteté improbable dans ce contexte météorologique... la scène entière enfin, empreinte d'une naïveté iconique qui rappelle pêle-mêle la $\mathrm{BD}$, une copie maladroite des tableaux apocalyptiques de Bosch ou les séries $\mathrm{B}$ de science-fiction, interdisent de voir là une véritable photographie. Cette (fausse) maladresse permet cependant de saisir d'une seul coup d'œil ce qui guide la réalisation de cette image: l'illustration des deux principaux mots du titre: «Tempête / Marée noire», (coiffés du surtitre, «La planète détraquée»). De ce point de vue, l'ensemble de la une fonctionne.

Il reste ce sentiment d'étrangeté d'un document qui se donne pour photographie et offre dans le même temps les indices évidents qu'il ne s'agit pas de cela. Le rapprochement avec la totalité des photos de ces trois journaux permet de comprendre de quoi est fait notre sentiment. Cette photo, la seule qui prétend montrer la tempête, n'en est pas une: c'est un montage s'affirmant comme tel. Une «photo juste» du météore, semble-t il être signifié, n'existe pas. En conséquence, en voici un succédané mais un succédané qui ne cherche pas à se 
faire passer pour ce qu'il n'est pas. Du combat contre la facilité, le journal sort vainqueur, il reste crédible, il ne nous aura pas trompés : la tempête, la «vraie», est définitivement, et justement, impossible à montrer.

\section{Quand ethos et pathos règlent le partage discursif de l'émotion}

Le premier est le garant de la crédibilité du journal qui, si elle est présupposée, reste toujours à réaffirmer. Or l'émotion n'est perçue comme telle qu'en raison même de la sincérité qu'on accorde à celui qui la vit ou prétend la faire partager: il faut que l'on croie à la sincérité de l'orateur - à son caractère moral ${ }^{14}$ - pour que l'on puisse croire à son émotion. Le second est ce par quoi le lecteur adhère au contrat de crédibilité. L'adhésion au discours de l'autre ne se construit pas sur la seule rationalité : elle résulte aussi d'une excitation des passions par le discours ${ }^{15}$. Dans ce mouvement, le lecteur, reconnaissant comme sincère l'émotion portée par le logos, la comprend, s'en saisit et la partage.

C'est à ces conditions seules que, entre les deux instances, l'émotion peut être portée par le logos. Cependant, le logos constitue toujours une mise à distance. Il permet de donner une forme organisée au mouvement de la conscience, qui avait, pour un temps, échappé à la pensée rationalisante. En effet, il intervient toujours après, dans un mouvement réflexif, alors que l'émotion est, au contraire, un mouvement continu en avant des mots, à l'image du sportif qui, en équilibre instable, file en glissant au sommet de la vague qui le porte, le pousse et pourtant jamais ne le rattrape (ou, quand elle le rattrape, c'est fini). Afin d'éluder le risque d'affaiblissement émotionnel, ces trois journaux font le choix d'une même stratégie: court-circuiter, dans le récit, le segment discursif central, taire l'évènement et affecter au discours la seule mission d'orienter l'éventuelle vague émotionnelle du lecteur. À partir de l'évanouissement narratif, les objets discursifs annexes contribuent à renforcer de deux façons la stratégie adoptée: d'une part, ils orientent la lecture vers le drame, d'autre part, ils contribuent au sentiment diffus d'inquiétude, terreau d'une émotion éventuelle.

Cette stratégie, si elle fonctionne, est mise au service d'une même visée. L'ensemble des articles procède à une ouverture au-delà de l'actualité. Tous inscrivent ces deux tempêtes dans une série beaucoup plus vaste et inquiétante d'évènements qui affectent l'équilibre écologique et dont l'homme est responsable sans pouvoir les maitriser. Ce processus d'élargissement et de désignation

14. Aristote, 1991, Rhétorique, Paris, Le Livre de Poche, p. 83.

15. Ibid. 
des responsabilités fait apparaitre où se trouve l'origine du mal, dans : «le développement de logiques propres qui échappent à l'humain [et qui] cumulent des risques chimique, nucléaire, génétique, climatique, alimentaire - qu'il faut désormais gérer ${ }^{16}$.

Ainsi se révèle le type d'émotion dont ces pages prétendent rendre compte: la vieille crainte de l'homme devant un cosmos à la logique impénétrable et menaçante, l'inquiétude panique des Grecs de l'Antiquité qui accompagne l'humanité depuis toujours. Cette émotion très ancienne, semblent dire ces articles, laisserait sans voix. Peut-être le silence est-il plus archaïque encore: pour éviter de provoquer à nouveau ces terrifiantes manifestations ne conviendrait-il pas de les taire? Peut-être. Il reste que l'émotion mise en scène à travers l'évanouissement narratif de l'objet même des articles s'inscrit dans une stratégie de discours fortement argumentative. Ainsi activée, l'émotion semble être utilisée comme déclencheur pédagogique pour une prise de conscience universelle dont la presse veut être l'instigatrice et la propagandiste. Telle est la posture ordinaire de la presse: son action, c'est l'action pédagogique, la seule que lui permettent son statut et son discours, la seule qui puisse authentifier son émotion. Jean-Pierre Chevènement, à la même époque, à propos de la marée noire provoquée par le pétrolier Erika, déclarait qu'il fallait «mettre l'émotion au service de la méthode ${ }^{17}{ }^{17}$. Le ministre de l'Intérieur traduisait politiquement ce qu'une partie de la presse avait, avant lui, construit médiatiquement.

16. Éditorial du supplément du Monde, 13 janvier 2000, p. II.

17. Le Monde, 19 janvier 2000, p. 6. 